\title{
3 Umgang mit Gesundheit und Krankheit
}

\author{
Jörg Kupfer
}

\begin{abstract}
Im Folgenden sollen einige geschlechtsspezifische Aspekte des Krankheits- und Gesundheitsverhaltens beschrieben werden. Während die Ebene des Krankheitsverhaltens, wie Symptomwahrnehmung und -bewertung oder das Inanspruchnahmeverhalten ärztlicher Hilfe eher geringere Geschlechtsunterschiede aufweist, zeigen sich im Gesundheitsverhalten, insbesondere bei dem Umgang mit Risikofaktoren oder einem primärpräventiven Verhalten deutliche Geschlechtsdifferenzen. Hieraus resultieren die Forderungen hinsichtlich einer stärkeren Berücksichtigung von geschlechtsspezifischen Bedürfnissen in der Primärprävention. Auch wenn bekannt ist, dass die im Moment vorhandenen Programme Männer weniger ansprechen, so gibt es aber noch wenig evidenzbasiertes Wissen dazu, wie entsprechende männerorientierte Programme aussehen müssten.
\end{abstract}

\subsection{Definitionen}

Zunächst sollen die Konstrukte Gesundheits- und Krankheitsverhaltens kurz erläutert werden. Die Begriffe unterscheiden sich aufgrund der An- oder Abwesenheit von Krankheitssymptomen. So wird der Begriff Gesundheitsverhalten dann verwandt, wenn keine Krankheitssymptome anwesend sind und Krankheitsverhalten meist bei bestehender Symptomatik. Die Konstrukte schließen einander aber nicht aus. So kann man auch typische Aspekte des Gesundheitsverhaltens zeigen, wenn man krank ist (Ernährung, körperliche Fitness etc.). Üblicherweise werden aber unterschiedliche Aspekte des Gesundheits- und Krankheitsverhaltens unter einem der genannten Konstrukte diskutiert.

Themen, die im Kontext dieses Artikels unter Krankheitsverhalten diskutiert werden:

- Symptomwahrnehmung und -bewertung

- Hilfesuchverhalten

Unter Gesundheitsverhalten versteht man all die Verhaltensweisen, die darauf abzielen die Gesundheit zu erhalten und das Risiko für Erkrankungen zu minimieren (Waller 2007). Themen die in diesem Kontext relevant sind, können unterschieden werden in: 
n gesundheitsförderliche Verhaltensweisen wie z.B.:

körperliche(s) Training/Fitness;

Wahrnehmung von Vorsorgeuntersuchungen

- und Vermeidung gesundheitsgefährtender Verhaltensweisen wie z.B.:

- Rauchen

- übermäßiger Alkohol- und Drogenkonsum

n Übergewicht

\subsection{Krankheitsverhalten}

\subsubsection{Symptomwahrnehmung und -bewertung}

Immer wieder wird berichtet, dass Männer Körpersymptome seltener wahrnehmen oder nicht als krankheitswertig einschätzen. Betrachtet man sich hierfür als einen Indikator die Einschätzung des subjektiven Gesundheitszustandes, so zeigt sich, dass dieser von Frauen und Männern sehr ähnlich beurteilt wird, auch wenn Männer seit vielen Jahren ihre Gesundheit geringfügig besser beurteilen als Frauen (Robert KochInstitut 2011). Ähnlich sind die Ergebnisse bei der Einschätzung der gesundheitsbezogenen Lebensqualität (Ellert et al. 2005).

Diese Einschätzungen könnten allerdings verwundern, wenn man bedenkt, dass die Lebenserwartung bei Geburt bei Frauen ca. 5 Jahre höher als bei Männern ist (Statistisches Bundesamt 2011a). Betrachtet man allerdings die beschwerdefreien Lebensjahre, so zeigen sich dort kaum Geschlechtsunterschiede (Jahr 2009: Männer 56,7 J. Frauen 57,7 J., Eurostat 2011). Dies bedeutet, dass die subjektiven Einschätzungen der Männer (bzgl. subjektiver Gesundheit und gesundheitsbezogener Lebensqualität) zumindest für weite Altersbereiche den objektiven Daten (beschwerdefreie Lebensjahre) nicht widersprechen .

Betrachtet man die Laienhypothesen von Männern und Frauen zu den unterschiedlichen Lebenserwartungen so spiegeln sie letztlich sozial unterschiedliche Rollenerwartungen wider (Frauen fokussieren eher auf die Rolle als Mutter und Sorgende für die Familie; Männer führen die geringere eigene Lebenserwartung eher auf ihre Rolle als Versorger der Familie zurück [Emslie u. Hunt 2008]). Genetische und biologische Faktoren, die in der wissenschaftlichen Diskussion als ebenso relevant erachtet werden, finden sich in den Laienhypothesen eher selten.

\subsubsection{Hilfesuchverhalten}

Auch das Inanspruchnahmeverhalten von ärztlicher Hilfe unterscheidet sich nicht fundamental zwischen den Geschlechtern. Auch wenn weniger Männer als Frauen im letzten Quartal zum Arzt gegangen sind ( $M=66 \%$ vs. $F=77 \%$ ), so zeigt sich in der Kontaktfrequenz $(M=3,6$ vs. $F=3,8)$ und bei den Krankenhausaufenthalten $(M=11 \%$ vs. $\mathrm{F}=13 \%$ im letzten Jahr) kaum ein Unterschied. Die beiden letztgenannten Indikatoren sind insofern relevant, als sich durch sie Hinweise auf eine Bestätigung folgender Hypothese finden müssten. Häufig wurde argumentiert, dass durch eine Fehleinschätzung von Körpersymptomen, Arztbesuche von Männern hinausgeschoben werden würden. Hierdurch könnte sich das Risiko für schwerwiegendere Diagnosen erhöhen. Dies müsste dann allerdings auch verknüpft sein mit einer höheren Kon- 
taktfrequenz des Arztes oder sich im Extremfall durch mehr Krankenhauseinweisungen zeigen. Auch beim letztgenannten Parameter „Anzahl der Nächte im Krankenhaus im letzten Jahr" ( $M=12 \mathrm{vs.} F=13$ im letzten Jahr) zeigen sich keine geschlechtsspezifischen Unterschiede (Statistisches Bundesamt et al. 2011).

\subsection{Gesundheitsverhalten}

\subsubsection{Körperliche(s) Training/Fitness}

Körperliche Fitness wirkt sich bekanntermaßen positiv auf zahlreiche Gesundheitsbereiche aus. Insofern könnte die wöchentliche sportlich aktive Zeit ein guter Indikator für gesundheitlich relevante Verhaltensweisen sein. Insbesondere bei den jüngeren Altersgruppen geben Männer häufiger und längere Zeiten für ihre sportliche Aktivitäten an als Frauen (s. Tab. 1, Robert-Koch Institut 2011). Im mittleren und hohen Erwachsenenalter scheint sich dieses Verhältnis zu verändern.

In der Gruppe ab dem 45. Lebensjahr gibt es deutlich mehr Männer als Frauen, die sportlich inaktiv sind. Bedenkt man, dass dieses Alter für viele chronische Erkrankungen einen riskanten Altersbereich darstellt, so könnte die nachlassende sportliche und körperliche Aktivität durchaus einen relevanten Risikofaktor für geschlechtsspezifische Inzidenzraten darstellen.

Tab. 1 Wöchentliche sportliche und körperliche Aktivität von Männern und Frauen (Daten aus: Robert Koch-Institut 2011)

\begin{tabular}{|c|c|c|c|}
\hline & kein Sport & $<2$ h Sport & $\geq 2$ h Sport \\
\hline \multicolumn{4}{|c|}{ Männer } \\
\hline $18-29$ & 15,4 & 14,5 & 70,1 \\
\hline $30-44$ & 33,0 & 21,3 & 45,7 \\
\hline $45-64$ & 40,1 & 17,5 & 42,4 \\
\hline$>64$ & 52,8 & 12,1 & 35,2 \\
\hline \multicolumn{4}{|l|}{ Frauen } \\
\hline $18-29$ & 21,8 & 23,4 & 54,9 \\
\hline $30-44$ & 34,7 & 24,8 & 40,4 \\
\hline $45-64$ & 33,8 & 22,3 & 43,8 \\
\hline$>64$ & 48,2 & 22,6 & 29,2 \\
\hline
\end{tabular}

\subsubsection{Wahrnehmung von Vorsorgeuntersuchungen}

Für Männer werden von den Krankenkassen Krebsvorsorgeuntersuchungen für verschiedene Krebsarten ab dem 45. Lebensjahr angeboten. In den Jahren 2000 bis 2008 lagen die Raten für die Inanspruchnahme dieser Untersuchungen bei Männern zwischen 18,3\% (2004) und 21,2\% (2008) bei den Frauen allerdings zwischen 46,8\% (2004) und 47,4\% (2008). Dieser Unterschied resultiert aber in erster Linie auf sehr hohen In- 
anspruchnahmeraten bei jüngeren Frauen (Altersgruppe 35-39 Jahre: 67,0\%). Im Alter nähern sich die Raten beider Geschlechter an (Altersgruppe 70-74 Jahre: $F=29,9 \%$, $M=27,3 \%$ ). Ab dem 75. Lebensjahr ist die Akzeptanz bei den männlichen Versicherten dann sogar höher als bei den weiblichen Versicherten (Altersgruppe 80 Jahre und älter: $\mathrm{F}=8,6 \%, M=18,0 \%)$. Interessanterweise unterscheiden sich die Inanspruchnahmeraten bei den Check-up Terminen nicht zwischen den Geschlechtern (im 2-JahresZeitraum: $F=41,0 \%, M=42,5 \%$ ) (Altenhofen o.J., Zentralinstitut für die kassenärztliche Versorgung 2009).

Bei der Inanspruchnahme primärpräventiver Programme zeigen sich allerdings drastische Unterschiede zwischen Frauen und Männern (s. Tab. 2). Diese Programme werden von Männern deutlich seltener angenommen, was zu der Forderung führte, dass diese oder ähnliche Angebot dringend geschlechtssensibel gestaltet werden müssen, um eine Chancengleichheit der Geschlechter zu erzielen (Kolip 2008).

Tab. 2 Inanspruchnahme primärpräventiver Programme 2009 (Daten aus: Robert Koch Institut 2011)

\begin{tabular}{|ccccc|}
\hline & Bewegung & Ernährung & Entspannung & mindestens ein Programm \\
\hline Männer & $8,7 \%$ & $4,1 \%$ & $3,0 \%$ & $11,1 \%$ \\
\hline Frauen & $16,7 \%$ & $6,0 \%$ & $6,3 \%$ & $20,8 \%$ \\
\hline
\end{tabular}

\subsubsection{Rauchen, Alkohol, Übergewicht}

Betrachtet man zuletzt die klassischen Risikofaktoren, so sieht man, dass Männer in allen 3 Bereichen eine ungünstigere Verhaltensweise an den Tag legen (s. Tab. 3). Lediglich im Bereich des Rauchverhaltens hat sich in den letzten Jahren eine Reduktion des Risikoverhaltens für Männer ergeben, während für das Übergewicht stetig steigende Zahlen berichtet werden. In einer kürzlich veröffentlichten Studie zeigte sich, dass die Geschlechtsunterschiede bei Kindern und Jugendlichen bezüglich Übergewicht und Adipositas eher gering sind. Allerdings ergaben sich teilweise deutliche Unterschiede hinsichtlich der Häufigkeit von Begleitsymptomen zwischen Jungen und Mädchen (Hypertonus, Hyperurikämie, HDL < $40 \mathrm{mg} / \mathrm{dl}$ häufiger bei Jungen, Wiegand 2007).

\section{Zusammenfassung}

Betrachtet man zunächst nochmals das Krankheitsverhalten von Männern auf der Basis von einer im Vergleich zu Frauen nahezu identischen Dauer der beschwerdefreien Lebensjahre, so erweisen sich die Unterschiede in der gesundheitsbezogenen Lebensqualität, im subjektiven Gesundheitsgefühl und auch im Inanspruchnahmeverhalten ärztlicher Leistungen zumindest für die Altersgruppe bis ca. 60 Jahre als wenig relevant für eine Erklärung der reduzierten Lebenserwartung von Männern. Interessanter könnten hier einige Aspekte des Gesundheitsverhaltens sein. Zum einen gibt es hier deutlichere Geschlechtsunterschiede und zum anderen könnten einige dieser Unterschiede insbesondere im höheren Alter Auswirkungen zeigen. Eine deutliche Reduktion der sportlichen Aktivität ab ca. dem 40. Lebensjahr, eine geringe Beteiligung an den Krebsvorsorgeuntersuchungen verbunden mit mehr Risikofaktoren wie Rauchen, riskantem Alkoholkonsum und einem hohen Anteil von Männern mit Übergewicht zeigen die potenziel- 
Tab. 3 Rauchverhalten, Alkoholkonsum und Übergewicht als klassische Verhaltensabhängige Risikofaktoren für die Entwicklung von Erkrankungen

\begin{tabular}{|c|c|c|}
\hline & Männer \% & Frauen $\%$ \\
\hline \multicolumn{3}{|l|}{ 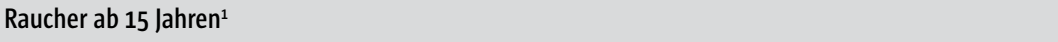 } \\
\hline 1995 & 36,0 & 21,6 \\
\hline 2005 & 34,4 & 23,4 \\
\hline 2009 & 33,2 & 22,3 \\
\hline \multicolumn{3}{|c|}{$\begin{array}{l}\text { Riskanter Alkoholkonsum } \\
\text { 12-Monats-Prävalenz, M > 24g, F > 12g Reinalkohol pro Tag }\end{array}$} \\
\hline 1995 & 26,8 & 15,3 \\
\hline 2005 & 19,2 & 14,2 \\
\hline 2009 & 18,1 & 14,1 \\
\hline \multicolumn{3}{|l|}{ Gewichtsverteilung BMI ${ }^{3}$} \\
\hline$<18,5$ & 0,7 & 3,4 \\
\hline $18,5-25$ & 39,2 & 53,7 \\
\hline $25-30$ & 44,4 & 29,1 \\
\hline$>30$ & 15,7 & 13,8 \\
\hline $\begin{array}{l}{ }^{1} \text { Daten aus: Statistisches Bundesamt 2011c } \\
{ }^{2} \text { Daten aus: Kraus et al. } 2010 \\
{ }^{3} \text { Daten aus: Statistisches Bundesamt 2011b }\end{array}$ & & \\
\hline
\end{tabular}

len Ansatzpunkte primärpräventiver Programme und nötiger Beratungsansätze auf. Da allerdings die im Moment angebotenen Programme die Männer nicht erreichen, sind hier neue Ansätze gefragt, die die spezifischen Bedürfnisse der Männer berücksichtigen. Hierbei müssen auch Aspekte wie traditionelle Männlichkeitsbilder, Anforderungen aus der geschlechtsspezifischen Arbeitsteilung, Unterschiede im kommunikativen Verhalten oder eine eher instrumentelle Einstellung zum eigenen Körper einbezogen werden (Brandes 2003; Dinges 2009; Zemp Stutz u. Ceschi 2007).

\section{Literatur}

Altenhofen L (o.J.) Hochrechnung zur Akzeptanz von Gesundheitsuntersuchungen und Krebsfrüherkennungsuntersuchungen bei gesetzlich Versicherten. http://www.zi-berlin.de/cms/fileadmin/images/content/PDFs alle/Akzeptanz_KFU_GU_FOBT.pdf, abgerufen am 28.10.2011

Brandes H (2003) Männlicher Habitus und Gesundheit. Blickpunkt Der Mann 1, 10-13

Dinges M (2009) Männer, die beratungsresistenten Gesundheitsidioten? Blickpunkt Der Mann 7, 19-23

Ellert U, Lampert T, Ravens-Sieberer U (2005) Messung der gesundheitsbezogenen Lebensqualität mit dem SF-8. Bundesgesundheitsblatt Gesundheitsforschung Gesundheitsschutz 48, 1330-1337

Emslie C, Hunt K (2008) The weaker sex? Exploring lay understandings of gender differences in life expectancy: A qualitative study. Soc Sci Med 67, 808-816

Eurostat (2011) Gesunde Lebensjahre bei der Geburt nach Geschlecht, http://epp.eurostat.ec.europa.eu/tgm/ refreshTableAction.do?tab=table\&plugin=1\&pcode=tsien180\&language=de, abgerufen am 28.10.2011

Kolip P (2008) Geschlechtergerechte Gesundheitsförderung und Prävention. Bundesgesundheitsblatt Gesundheitsforschung Gesundheitsschutz 51, 28-35 
Kraus L, Pabst A, Piontek D, Müller S (2010) Kurzbericht Epidemiologischer Suchtsurvey. Tabellenband: Trends der Prävalenz von Alkoholkonsum, episodischem Rauschtrinken und problematischem Alkoholkonsum nach Geschlecht und Alter 1995-2009, http://www.ift.de/index.php?id=410, abgerufen am 28.10.2011

Robert Koch-Institut (Hrsg.) (2011) Beiträge zur Gesundheitsberichterstattung des Bundes. Daten und Fakten: Ergebnisse der Studie "Gesundheit in Deutschland aktuell 2009", Berlin

Statistisches Bundesamt (2011a) Bevölkerung und Erwerbstätigkeit. Sterbetafel Deutschland, Wiesbaden. Erschienen 20.9.2011, http://www.destatis.de/jetspeed/portal/cms/Sites/destatis/Internet/DE/Content/ Statistiken/Bevoelkerung/GeburtenSterbefaelle/Tabellen/Content100/SterbetafelDeutschland,property= file.xls, abgerufen am 28.10.2011

Statistisches Bundesamt (2011b) Mikrozensus - Fragen zur Gesundheit - Körpermaße der Bevölkerung. Wiesbaden: Statistisches Bundesamt, http://www.destatis.de/jetspeed/portal/cms/Sites/destatis/Internet/DE/ Content/Publikationen/Fachveroeffentlichungen/Gesundheit/Gesundheitszustand/Koerperma_C3_9Fe, templateld=renderPrint.psml, abgerufen am 28.10.2011

Statistisches Bundesamt (2011c) Mikrozensus - Fragen zur Gesundheit - Rauchgewohnheiten der Bevölkerung. Wiesbaden: Statistisches Bundesamt, http://www.destatis.de/jetspeed/portal/cms/Sites/destatis/Internet/ $\mathrm{DE} /$ Content/Publikationen/Fachveroeffentlichungen/Gesundheit/Gesundheitszustand/Rauchgewohnheiten, templateld=renderPrint.psml, abgerufen am 28.10.2011

Statistisches Bundesamt (Destatis), Wissenschaftszentrum Berlin für Sozialforschung (WZB),in Zusammenarbeit mit Das Sozio-oekonomische Panel (SOEP) am Deutschen Institut für Wirtschaftsforschung (DIW)(Hrsg.) (2011) Datenreport 2011. Ein Sozialbericht für die Bundesrepublik DeutschlandBand. Bonn: Bundeszentrale für politische Bildung/bpb

Waller H (2007) Sozialmedizin - Grundlagen und Praxis, 6., überarbeitete und erweiterte Auflage. Stuttgart: Verlag Kohlhammer

Wiegand S (2007) Geschlecht und Adipositas - bedeutet Dicksein dasselbe für Jungen und Mädchen? Ther Umsch 64, 319-324

Zemp Stutz E, Ceschi M (2007) Geschlecht und Kommunikation im ärztlichen Setting - eine Übersicht. Ther Umsch 64, 331-335

Zentralinstitut für die kassenärztliche Versorgung (2009) Teilnahme an gesetzlichen Früherkennungsuntersuchungen und an Beratungen zur Prävention des Darmkrebs (in Prozent der anspruchsberechtigten Altersgruppe) im Jahr 2008 (Bundesgebiet), Köln. http://www.zi-berlin.de/cms/fileadmin/images/content/PDFs_ alle/Beteiligung_Frueherk_2008.pdf (letzte Aktualisierung 21.1.2010) 